\title{
Erratum
}

\section{Correlated One-Center Wavefunctions for Two-Electron Molecules}

\author{
II. Configuration-Interaction Functions and Application to $\mathrm{HeH}^{+}$ \\ F. GReIN and T.-J. TsENG \\ Theoret. chim. Acta (Berl.) 12, 57-65 (1968)
}

Received September 8, 1969

A computer programming mistake was found. Tables $2,3,4$, and 5 have been recalculated and are reproduced in full for the sake of completeness. The variation of $\alpha$ was performed uniformly in steps of 0.01 , the variation of the orbital exponents (Tables 3 and 5 ) in steps of 0.01 or less.

Table 2. Ground state energies (in a.u.) of $\mathrm{HeH}^{+}$, obtained for fixed orbital exponents at $R=1.4$ a.u.

\begin{tabular}{rlllll}
\hline$m$ & $\alpha_{\mathrm{I}}$ & $-E_{\mathrm{I}}^{0}$ & $-E_{\mathrm{I}}^{\alpha}$ & $\Delta E_{\mathrm{I}}$ & $-\Delta E_{\mathrm{I}} / E_{\mathrm{I}}^{0} \%$ \\
\hline 1 & 0.40 & 2.76506 & 2.84792 & 0.08286 & 3.00 \\
3 & 0.36 & 2.80045 & 2.85002 & 0.04957 & 1.77 \\
6 & 0.29 & 2.82832 & 2.85338 & 0.02506 & 0.89 \\
7 & 0.30 & 2.85734 & 2.88414 & 0.02680 & 0.94 \\
8 & 0.27 & 2.89073 & 2.91394 & 0.02321 & 0.80 \\
9 & 0.25 & 2.89811 & 2.91768 & 0.01937 & 0.68 \\
10 & 0.25 & 2.91563 & 2.93570 & 0.02007 & 0.69 \\
11 & 0.23 & 2.92515 & 2.94243 & 0.01728 & 0.59 \\
12 & 0.23 & 2.93313 & 2.95055 & 0.01742 & 0.59 \\
13 & 0.23 & 2.93786 & 2.95415 & 0.01629 & 0.55 \\
14 & 0.22 & 2.94083 & 2.95419 & 0.01336 & 0.45 \\
15 & 0.15 & 2.95069 & 2.95478 & 0.00409 & 0.14 \\
17 & 0.14 & 2.95266 & 2.95522 & 0.00256 & 0.09 \\
18 & 0.14 & 2.95650 & 2.95917 & 0.00267 & 0.09 \\
19 & 0.18 & 2.95931 & 2.96343 & 0.00412 & 0.14 \\
20 & 0.17 & 2.96013 & 2.96364 & 0.00351 & 0.12 \\
\hline
\end{tabular}

Table 3. Ground state energies (in a.u.) of $\mathrm{HeH}^{+}$, obtained for optimized orbital exponents at $R=1.4$ a.u.

\begin{tabular}{llllllll}
\hline$m$ & $-E_{\mathrm{II}}^{\mathrm{O}}$ & $-E_{\mathrm{II}}^{\alpha^{*}}$ & $-E_{\mathrm{II}}^{\alpha}$ & $\Delta E_{\mathrm{II}}$ & $-\frac{\Delta E_{\mathrm{II}}}{E_{\mathrm{II}}^{0}} \%$ & $E_{\mathrm{II}}^{\alpha^{*}}-E_{\mathrm{II}}^{\alpha}$ & $-\frac{E_{\mathrm{II}}^{*^{*}}-E_{\mathrm{II}}^{\alpha}}{E_{\mathrm{II}}^{\alpha^{*}}} \%$ \\
\hline & & & & & & & \\
1 & 2.80735 & 2.83065 & 2.84852 & 0.04117 & 1.47 & 0.01787 & 0.63 \\
6 & 2.82657 & 2.84693 & 2.85293 & 0.02636 & 0.93 & 0.00600 & 0.21 \\
7 & 2.82851 & 2.85234 & 2.85358 & 0.02507 & 0.89 & 0.00124 & 0.04 \\
8 & 2.89469 & 2.91243 & 2.91484 & 0.02015 & 0.70 & 0.00241 & 0.08 \\
& 2.89769 & 2.91678 & 2.91765 & 0.01996 & 0.69 & 0.00087 & 0.03
\end{tabular}

